\title{
Interaction between surfaces with solvophobicity or solvophilicity immersed in solvent: Effects due to addition of solvophobic or solvophilic solute
}

\author{
$\operatorname{AUTHOR}(\mathrm{S})$ : \\ Kinoshita, M
}

\section{CITATION:}

Kinoshita, M. Interaction between surfaces with solvophobicity or solvophilicity immersed in solvent: Effects due to addition of solvophobic or solvophilic solute. JOURNAL OF CHEMICAL PHYSICS 2003, 118(19): 8969-8981

\section{ISSUE DATE:}

2003-05-15

URL:

http://hdl.handle.net/2433/50433

\section{RIGHT:}

Copyright 2003 American Institute of Physics. This article may be downloaded for personal use only. Any other use requires prior permission of the author and the American Institute of Physics. 


\title{
Interaction between surfaces with solvophobicity or solvophilicity immersed in solvent: Effects due to addition of solvophobic or solvophilic solute
}

\author{
Masahiro Kinoshita ${ }^{a)}$ \\ Institute of Advanced Energy, Kyoto University, Uji, Kyoto 611-0011, Japan
}

(Received 14 November 2002; accepted 20 February 2003)

\begin{abstract}
Integral equation theories with bridge functions incorporated in the closure equations are employed to analyze how the solvent-induced interaction between surfaces is influenced by solute addition to the solvent. The solvent particles interact through a hard-core plus attractive potential. The surfaces are solvophobic or solvophilic, and the solute has rather high solvophobicity or solvophilicity: A total of four combinations of the surface and solute properties are considered. The solute addition always leads to a downward shift, a shift in a more attractive direction, of the surface interaction (except at very small surface separations). The shift becomes more pronounced as the solute solvophobicity or solvophilicity increases and the solute concentration becomes higher. Overall, the solute effects are the smallest when the solute is neither solvophobic nor solvophilic. The physical origins of the shift are discussed in detail by relating the interaction to the structure of the solventsolute mixture confined between two surfaces. (C) 2003 American Institute of Physics.
\end{abstract}

[DOI: $10.1063 / 1.1566935]$

\section{INTRODUCTION}

The interaction between surfaces (or macroparticles) immersed in solvent is a central issue in colloidal science and biophysics, and density functional and integral equation theories are very useful for elucidating the solvent-mediated interaction at a microscopic level. In recent theoretical studies, the simplest model based on the hard-core repulsion was frequently employed. ${ }^{1-10}$ Although the model is of fundamental importance in exclusive investigation of the entropic excluded-volume effects, attractive parts of the potentials also play essential roles and can never be neglected. ${ }^{11-21}$ The effects due to the solvent-solvent and surface-solvent potentials have been analyzed, and a significant amount of information is already available. ${ }^{3,13-15}$ However, the important subject, understanding how the solute added to the solvent modifies the surface interaction, has not yet been considered in detail. The most popular solvent is water, and it is very interesting to ask how the interaction between surfaces with hydrophobicity or hydrophilicity is influenced by hydrophobic or hydrophilic solute added to water. There are a total of four combinations of the surface and solute properties in terms of the affinity with water. Effects of highly hydrophobic solutes on the interaction between hydrophobic surfaces in water were analyzed by the author ${ }^{17-20}$ using simple model systems, but the other three combinations remain to be treated. In biological systems, for example, the interactions between macromolecules and those between a macromolecule and a membrane are induced not in pure water but in aqueous solution containing a variety of solute molecules.

The present article contributes to theoretical elucidation of the solute effects on the surface interaction. A simple

${ }^{\text {a)} F A X: ~+81-774-38-3508 . ~ E l e c t r o n i c ~ m a i l: ~ k i n o s h i t @ i a e . k y o t o-u . a c . j p ~}$ model of spherical particles is employed for solvophobic or solvophilic surfaces in solvent containing solvophobic or solvophilic solute. The singlet Ornstein-Zernike (OZ) approach is used to calculate the structure of the solvent-solute mixture near a single surface and the surface interaction induced. The reference interaction site model (RISM) theory ${ }^{22}$ is also utilized to analyze the structure of the mixture confined between two surfaces as a function of the surface separation. A useful method of estimating the bridge functions to be incorporated in the closure equations is proposed for a system comprising particles with extremely high size asymmetry. The affinity of the solute or the surface with the solvent, the solute size, and the solute concentration are considered as major parameters.

\section{MODEL AND THEORY}

\section{A. Model potentials}

The present model system is chosen to roughly mimic hydrophobic or hydrophilic surfaces immersed in water containing hydrophobic or hydrophilic solute. It is assumed that small spheres with diameter $d_{\mathrm{S}}$ form the solvent. The solute molecules are modeled as medium-sized spheres with diameter $d_{\mathrm{M}}$, and three different values larger than $d_{\mathrm{S}}\left(d_{\mathrm{M}}\right.$ $=2 d_{\mathrm{S}}, 4 d_{\mathrm{S}}$, and $6 d_{\mathrm{S}}$ ) are considered for $d_{\mathrm{M}}$. This is because in biological systems the solute molecules are usually much smaller than macromolecules but larger than water molecules. The surface is treated as a sufficiently large sphere with small curvature that is present in the solventsolute mixture at infinite dilution. The diameter of the large sphere $d_{\mathrm{L}}$ is set at $30 d_{\mathrm{S}}$. The subscripts, "S," "M," and "L" represent "small," "medium-sized," and "large," respectively. The solvent-solvent (S-S), solute-solvent (M-S), and solute-solute $(\mathrm{M}-\mathrm{M})$ potentials are expressed by 


$$
\begin{aligned}
& u_{i j}(r)=\infty \quad \text { for } r<d_{i j}, \\
& u_{i j}(r)=-4 \varepsilon_{i j}\left(d_{i j} / r\right)^{6} \text { for } r>d_{i j}, \\
& d_{i j}=\left(d_{i}+d_{j}\right) / 2, \quad i, j=\mathrm{S}, \mathrm{M} .
\end{aligned}
$$

The depth of the attractive potential is $4 \varepsilon_{i j}$. Setting $\varepsilon_{i j}$ at zero yields the hard-core potential. The distance between centers of the two particles considered is always denoted by $r$. The surface-solvent $(\mathrm{L}-\mathrm{S})$ and surface-solute $(\mathrm{L}-\mathrm{M})$ potentials are assumed to have the form ${ }^{13,14}$

$u_{\mathrm{L} i}(r)=\infty$ for $r<d_{\mathrm{L} i}$,

$u_{\mathrm{L} i}=-\lambda_{\mathrm{L} i}\left(d_{\mathrm{L} i} / r\right) \exp \left\{-\kappa_{\mathrm{L} i}\left(r / d_{\mathrm{L} i}-1\right)\right\} \quad$ for $\quad r>d_{\mathrm{L} i}$,

$d_{\mathrm{L} i}=\left(d_{\mathrm{L}}+d_{i}\right) / 2, \quad i=\mathrm{S}, \mathrm{M}$,

in which $\lambda_{\mathrm{L} i}$ and $\kappa_{\mathrm{L} i}$ control the depth and the range of the attractive tail, respectively. The hard-core potential is obtained by setting $\lambda_{\mathrm{L} i}$ at zero.

Unless otherwise mentioned, the solvent particles interact through a hard-core plus attractive potential. When the solute is polar, they are solvophilic and the $\mathrm{M}-\mathrm{S}$ and $\mathrm{M}-\mathrm{M}$ potentials include sufficiently strong attractions. No attractions (or only weak attractions) are included in these potentials for a nonpolar, solvophobic solute. The most important parameter is $\varepsilon_{\mathrm{MS}}$ representing the polarity of the solute. With a large value of $\varepsilon_{\mathrm{MS}}$, for instance, the solute is solvophilic and as $\varepsilon_{\mathrm{MS}}$ becomes smaller the solvophilicity decreases. When the surface is solvophilic, it is charged and attraction arises in the $\mathrm{L}-\mathrm{S}$ potential. There is no attractive tail in the potential between a solvophobic surface and the solvent. In the $\mathrm{L}-\mathrm{M}$ potential, attraction is included only when both the surface and the solute are solvophilic.

In most of the analyses the solute has rather high solvophobicity or solvophilicity. The total packing fraction of the solvent-solute system, $\eta_{\mathrm{S}}+\eta_{\mathrm{M}}\left(\eta_{i}=\pi \rho_{i} d_{i}^{3} / 6 ; \rho_{i}\right.$ is the number density), is fixed at 0.383 , and $\eta_{\mathrm{M}}$ considered is in the range $0.001 \leqslant \eta_{\mathrm{M}} \leqslant 0.120$. This corresponds to 24000 $\geqslant \rho_{\mathrm{S}} / \rho_{\mathrm{M}} \geqslant 140$ in the case of $d_{\mathrm{M}}=4 d_{\mathrm{S}}$ : The solute concentration considered is significantly low. $4 \beta \varepsilon_{\mathrm{SS}}$ ( $\beta$ has the usual meaning) is set at 1.0 , and $\varepsilon_{\mathrm{MM}}$ is set equal to $\varepsilon_{\mathrm{MS}}$. When the solute is highly solvophobic, the bulk mixture undergoes phase separation into two immiscible liquids in a certain composition range. In such cases the solute concentration $\eta_{\mathrm{M}}$ is set sufficiently smaller than the spinodal value $\eta_{\mathrm{M}}^{*}$ beyond which the bulk mixture cannot exist as a single phase even in a metastable state $\left[\mathrm{D}(0) \rightarrow+0\right.$ as $\eta_{\mathrm{M}} \rightarrow \eta_{\mathrm{M}}^{*}$ -0 , where $\mathrm{D}(0)$ denotes the denominator of the $\mathrm{OZ}$ equation in the Fourier space at zero wave number $\left.{ }^{17}\right]$.

\section{B. Integral equation theories}

The singlet $\mathrm{OZ}$ equation is coupled with the closure equation in which a bridge function is incorporated. The bridge function is estimated in accordance with the procedure described in the next section. It should be noted that the singlet $\mathrm{OZ}$ equation is exact and only the bridge function includes an approximation. ${ }^{2}$ The interaction induced between surfaces (large spheres) $\Phi_{\mathrm{LL}}(r)$ is discussed in terms of the potential of mean force. The calculation process ${ }^{3,15-18}$ comprises three steps:

(1) Calculate the $\mathrm{S}-\mathrm{S}, \mathrm{M}-\mathrm{S}$, and $\mathrm{M}-\mathrm{M}$ correlation functions.

(2) Calculate the $\mathrm{L}-\mathrm{S}$ and $\mathrm{L}-\mathrm{M}$ correlation functions. The reduced density profiles of the solvent and solute particles near a single surface (the surface-solvent and surface-solute pair distribution functions) $g_{\mathrm{L} i}(r)(i=\mathrm{S}, \mathrm{M})$ are obtained in this step.

(3) Determine the L-L correlation functions and obtain the potential of mean force (the solvent-induced interaction) $\Phi_{\mathrm{LL}}(r)$. The potential acting directly between the surfaces is not considered.

In the numerical solution of the basic equations, the grid width $\delta r$ and the number of grid points $N$ are set at $0.01 d_{\mathrm{S}}$ and 16384 , respectively.

In the calculation summarized above, one cannot gain access to the structure of the solvent-solute mixture confined between two surfaces. Therefore, the RISM theory ${ }^{19-22}$ is employed to complement the calculation. A pair of large spheres, the surface separation of which is $L\left(=r-d_{\mathrm{L}}\right)$, is treated as a supermolecule with two sites immersed in the mixture at infinite dilution. The bridge functions obtained by the procedure described in the next section are incorporated in the closure equations. Let $G_{\mathrm{Li}}(L ; r)$ be the pair distribution function between one of the large spheres and a solvent particle or a solute particle. It is obvious that

$$
G_{\mathrm{L} i}(\infty ; r)=g_{\mathrm{L} i}(r), \quad i=\mathrm{S}, \mathrm{M} .
$$

Information on the structure of the mixture confined between two surfaces is contained in

$$
F_{\mathrm{L} i}(L ; r)=G_{\mathrm{L} i}(L ; r)-g_{\mathrm{L} i}(r) .
$$

Further, the author introduces $\Delta\left(G_{\mathrm{LS}}(L ; r)-g_{\mathrm{LS}}(r)\right)$ defined by

$$
\begin{aligned}
\Delta\left(G_{\mathrm{LS}}(L ; r)-g_{\mathrm{LS}}(r)\right) & \\
= & {\left[{ }^{\prime \prime} G_{\mathrm{LS}}(L ; r)-g_{\mathrm{LS}}(r), \text { in solvent-solute mixture }\right] } \\
& -\left[{ }^{\prime \prime} G_{\mathrm{LS}}(L ; r)-g_{\mathrm{LS}}(r), \text { in pure solvent }\right] .
\end{aligned}
$$

The Fourier transform of $F_{\mathrm{L} i}(L ; r)$ at zero wave number is denoted by $\Lambda_{i}(L)$. This parameter represents the strength of the surface-solvent or surface-solute correlation. ${ }^{19-21}$

\section{Bridge functions}

It was shown that the functional form of the bridge function suited to hard spheres is applicable to any of dense fluids whose short-range structures are determined mainly by the repulsive part of the interaction potential. ${ }^{23}$ In fact, the hardsphere bridge functions have been applied successfully to polar fluids interacting through strongly attractive potentials $^{24-27}$ as well as to nonpolar fluids. Among various functional forms, the semiempirical bridge function proposed for hard-sphere fluids by Verlet ${ }^{28}$ is very simple and convenient. It relates the bridge function $b(r)$ to the correlation function $\gamma(r)=h(r)-c(r)$ ( $h$ and $c$ denote, respectively, the total and direct correlation functions) simply by 


$$
b(r)=-0.5\{\gamma(r)\}^{2} /\{1+0.8 \gamma(r)\} .
$$

Equation (6) was also extended to molecular hard-body fluids and the results obtained were quite accurate. ${ }^{29-32}$ However, Eq. (6) has a singularity at $\gamma=-1 / 0.8$ causing serious errors for mixtures of Lennard-Jones (LJ) particles with relatively high size asymmetry. To overcome this problem, Duh and Henderson ${ }^{33}$ proposed an alternative method expressed as

$b_{i j}(r)=-0.5\left\{\gamma_{i j}(r)\right\}^{2} /\left\{1+0.8 \gamma_{i j}(r)\right\} \quad$ for $\quad \gamma_{i j}(r)>0$,

$b_{i j}(r)=-0.5\left\{\gamma_{i j}(r)\right\}^{2}$ for $\gamma_{i j}(r)<0$.

Equation (7) and its first and second derivatives with respect to $\gamma_{i j}$ are continuous at $\gamma_{i j}=0$.

The mixtures treated in the present article, however, have extremely high size asymmetry: The author has found that $\gamma_{i j}(r)$ takes very large, negative values at significantly many separations and employing Eq. (7b) gives rise to pathological (abnormally large) $b_{i j}(r)$ at these separations for the $\mathrm{L}-\mathrm{M}$ and $\mathrm{L}-\mathrm{L}$ pairs. ${ }^{10}$ Therefore, Eq. (7b) is replaced by another equation and the resultant method is expressed as

$b_{i j}(r)=-0.5\left\{\gamma_{i j}(r)\right\}^{2} /\left\{1+0.8 \gamma_{i j}(r)\right\} \quad$ for $\gamma_{i j}(r)>0$,

$b_{i j}(r)=-0.5\left\{\gamma_{i j}(r)\right\}^{2} /\left\{1-0.8 \gamma_{i j}(r)\right\} \quad$ for $\gamma_{i j}(r)<0$.

Equation (8) and its first and second derivatives with respect to $\gamma_{i j}$ are also continuous at $\gamma_{i j}=0$.

For highly asymmetrical hard-sphere mixtures, Attard and Patey $^{2}$ developed the so-called (HNC-Padé) theory. In this theory all the bridge diagrams with two and three field points are exactly calculated using Monte Carlo integration techniques and those with more than three are included by the Padé approximant. For the interaction induced between large hard spheres in small hard spheres $\left(d_{\mathrm{L}} / d_{\mathrm{S}}=10\right.$ and $\left.\eta_{\mathrm{S}}=0.314\right)$ for which computer simulation data ${ }^{7,34}$ is available, the following three methods are tested. In method 1 , Eq. (8) is used for all $b_{i j}(r)$ including $b_{\mathrm{LL}}(r)$. In method 2, $b_{\mathrm{LL}}(r)$ is obtained from the HNCP theory and $b_{i j}(r)$ for all the other pairs are calculated using Eq. (8). In method 3, $b_{\mathrm{LL}}(r)$ is taken to be the average of the two $\mathrm{L}-\mathrm{L}$ bridge functions from methods 1 and 2 and $b_{i j}(r)$ for all the other pairs are calculated using Eq. (8). While results from the three methods are quantitatively similar, the result from method 3 is the most accurate. It is compared with the computer simulation data in Fig. 1, indicating an excellent agreement. Method 3 is employed throughout the present study. Since $\rho_{\mathrm{M}} / \rho_{\mathrm{S}}$ is extremely small and $d_{\mathrm{M}} / d_{\mathrm{S}}$ is not very large, the system in which large spheres are immersed is treated as a single-component system of small spheres with $\eta_{\mathrm{S}}$ $=0.383$ when the HNCP theory is used.

The calculation using the HNCP theory is the most timeconsuming part of the proposed method. However, if a bridge function $b_{\mathrm{LL} 1}(r)$ calculated under one condition $\left(d_{\mathrm{L}}\right.$ $=d_{\mathrm{L} 1}$ and $\left.\eta_{\mathrm{S}}=\eta_{\mathrm{S} 1}\right)$ is available, the function $b_{\mathrm{LL} 2}(r)$ under another condition $\left(d_{\mathrm{L}}=d_{\mathrm{L} 2}\right.$ and $\left.\eta_{\mathrm{S}}=\eta_{\mathrm{S} 2}\right)$ can be estimated from

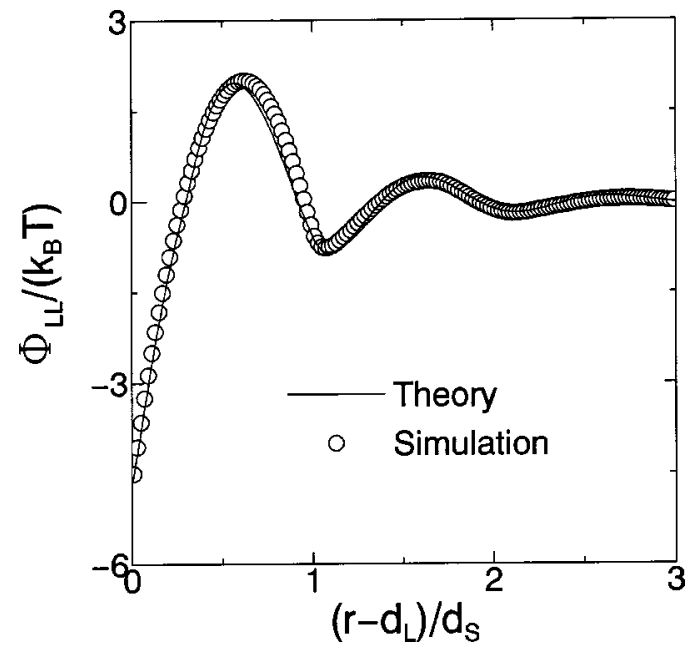

FIG. 1. Interaction between large hard spheres theoretically calculated using method $3\left(d_{\mathrm{L}} / d_{\mathrm{S}}=10\right.$ and $\left.\eta_{\mathrm{S}}=0.314\right)$. The force obtained in the computer simulation of Biben et al. (Ref. 34) was smoothed and integrated by Roth et al. (Ref. 7), yielding the simulation data compared with the theoretical result.

$$
b_{\mathrm{LL} 2}(r)=b_{\mathrm{LL} 1}(r)\left(d_{\mathrm{L} 2} / d_{\mathrm{L} 1}\right)\left(\eta_{\mathrm{S} 2} / \eta_{\mathrm{S} 1}\right)^{2} .
$$

The factor $d_{\mathrm{L} 2} / d_{\mathrm{L} 1}$ comes from the Derjaguin approximation, ${ }^{35}$ and the factor $\left(\eta_{\mathrm{S} 2} / \eta_{\mathrm{S} 1}\right)^{2}$ is based on the fact that the leading bridge diagram depends on the squared number density. ${ }^{2}$ The result from Eq. (9) is fairly accurate as long as $\eta_{\mathrm{S} 2}$ is not far from $\eta_{\mathrm{S} 1}$.

\section{SURFACE INTERACTION IN PURE SOLVENT}

The small spheres are referred to as solvent particles. The case where no solute is added to the solvent is labeled as case 0 . Three subcases of case 0 (cases $0-1,0-2$, and $0-3$ ) are considered and the parameters for these subcases are set as summarized in Table I. The interaction between large hard spheres in the solvent of small hard spheres (case 0-1: no attractions are included in the potentials) is induced by the entropic excluded-volume effects alone and shown in Fig. 2. When the attractive potential is introduced between small spheres only (case 0-2), the large-sphere surface becomes solvophobic and as observed in Fig. 2 the interaction $\Phi_{\mathrm{LL}}$ shifts in a significantly more attractive direction. It is much less oscillatory and attractive at all separations. The interaction between hydrophobic surfaces (macroparticles) in waterlike fluids with more realistic models exhibits similar characteristics. ${ }^{15,16}$ With further introduction of the surfacesolvent attraction (case 0-3), the surface becomes solvophilic and $\Phi_{\mathrm{LL}}$ shifts in a considerably more repulsive direc-

TABLE I. Parameters set in the three subcases of case 0 : cases $0-1,0-2$, and $0-3$ (in case 0 , no solute is added to the solvent). In case $0-3, \kappa_{\mathrm{LS}}=5.0$. The common parameters to all of these subcases are $\eta_{\mathrm{M}}=0.0$ and $\eta_{\mathrm{S}}=0.383$.

\begin{tabular}{ccc}
\hline \hline Case & $4 \beta \varepsilon_{\mathrm{SS}}$ & $\beta \lambda_{\mathrm{LS}}$ \\
\hline $0-1$ & 0.0 & 0.0 \\
$0-2$ & 1.0 & 0.0 \\
$0-3$ & 1.0 & 0.5 \\
\hline \hline
\end{tabular}




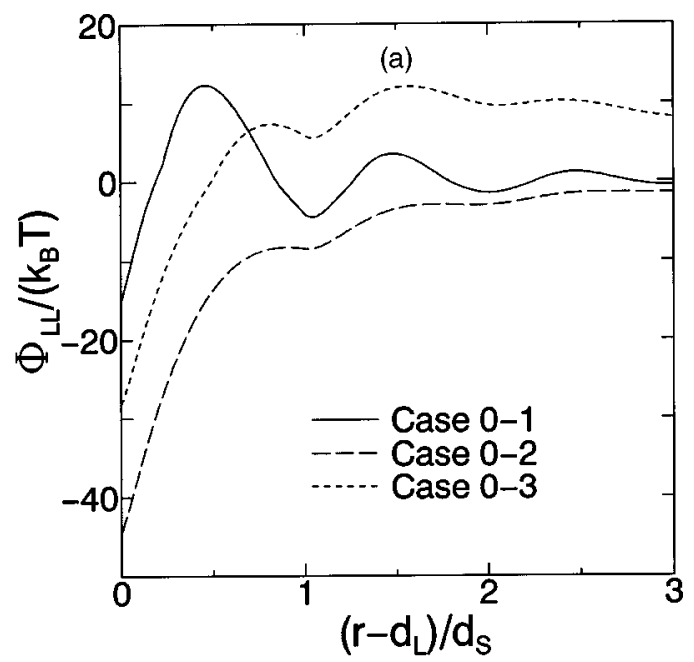

TABLE II. Parameters set in the four subcases of case 1: cases 1-1, 1-2, 1-3, and 1-4 (in case 1, both the surface and the solute are solvophobic). The common parameters to all of these subcases are $\eta_{\mathrm{S}}+\eta_{\mathrm{M}}=0.383,4 \beta \varepsilon_{\mathrm{SS}}$ $=1.0, \varepsilon_{\mathrm{MM}}=\varepsilon_{\mathrm{MS}}, \lambda_{\mathrm{LS}}=0.0$, and $\lambda_{\mathrm{LM}}=0.0$.

\begin{tabular}{cccc}
\hline \hline Case & $d_{\mathrm{M}} / d_{\mathrm{S}}$ & $\eta_{\mathrm{M}}$ & $4 \beta \varepsilon_{\mathrm{MS}}$ \\
\hline $1-1$ & 2.0 & 0.010 & 0.0 \\
$1-2$ & 2.0 & 0.050 & 0.0 \\
$1-3$ & 4.0 & 0.001 & 0.0 \\
$1-4$ & 6.0 & 0.001 & 0.2 \\
\hline \hline
\end{tabular}

$\sim 1$, however, the small spheres are densely packed within the confined domain]. The repulsive component generally dominates and the induced force is more repulsive as compared to the force in case 0-2. The upward shift of $\Phi_{\mathrm{LL}}$ mentioned in the last paragraph can thus be explained.

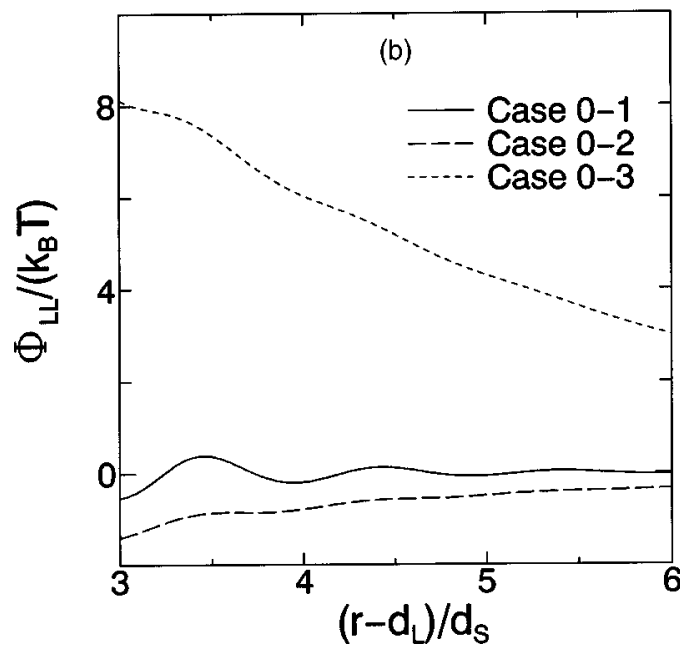

FIG. 2. Interaction between surfaces (large spheres) immersed in pure solvent of small spheres: $0 \leqslant\left(r-d_{\mathrm{L}}\right) / d_{\mathrm{S}} \leqslant 3$ (a) and $3 \leqslant\left(r-d_{\mathrm{L}}\right) / d_{\mathrm{S}} \leqslant 6$ (b). See Table I for the definition of cases $0-1,0-2$, and 0-3.

tion: It is repulsive except at very small separations. Again, the interaction between weakly charged surfaces in the water-like fluids has similar characteristics. ${ }^{15}$

The induced force $F_{\mathrm{LL}}(r)=-d \Phi_{\mathrm{LL}}(r) / d r$ arises from thermal pressure due to the small spheres acting on the largesphere surfaces. ${ }^{1,8-10,36}$ When the attractions are incorporated in the potentials between small spheres as in case 0-2, a new factor comes into play in addition to the entropic excludedvolume effects: Since the surface is solvophobic, at sufficiently small surface separations, the density of small spheres within the domain confined between two surfaces (particularly near the surfaces) is driven to be lower than the density near a single surface. The thermal pressure contributing to the net force as a repulsive component becomes lower than the pressure contributing as the attractive component. Consequently, the induced force generally becomes more attractive than in case $0-1$ and $\Phi_{\mathrm{LL}}$ exhibits the downward shift mentioned in the last paragraph. When the surface is made solvophilic as in case $0-3$, the small-sphere density near the surfaces within the confined domain is driven to be higher than near a single surface. This holds true except at the small surface separations $\left(r-d_{\mathrm{L}}\right) / d_{\mathrm{S}}<2\left[\right.$ at $\left(r-d_{\mathrm{L}}\right) / d_{\mathrm{S}}$

\section{SURFACE INTERACTION IN SOLVENT-SOLUTE MIXTURE}

\section{A. Solvophobic surface and solvophobic solute (case 1)}

The case where both the surface and the solute are solvophobic is labeled as case 1 . Four subcases of case 1 (cases $1-1,1-2,1-3$, and 1-4) are considered and the values given to the parameters for these subcases are collected in Table II. Effects of adding a solute with considerably high solvophobicity (the medium-sized spheres are referred to as solute particles) on the interaction between solvophobic surfaces are illustrated in Fig. 3. In the present model, as $d_{\mathrm{M}}$ increases with $\varepsilon_{\text {MS }}$ fixed at zero, the solvation free energy and solvophobicity of the solute become progressively higher. When the $\varepsilon_{\mathrm{MS}}$ value is changed to a nonzero, small one with fixing $d_{\mathrm{M}}$, the solute becomes less solvophobic. The presence of solute particles with $d_{\mathrm{M}}=2 d_{\mathrm{S}}$ in a low concentration (case 1-1) causes only a slight shift of the surface interaction $\Phi_{\mathrm{LL}}$ in a more attractive direction. Increasing the concentration (case 1-2: $\eta_{\mathrm{M}}=0.050$ ), however, leads to a more pronounced shift (in a more attractive direction except at very small separations) and a longer interaction range. In cases of the highly solvophobic solutes with the larger sizes (cases 1-3 and 1-4), the concentration must be set at a much lower value to avoid the phase separation in the bulk (the spinodal concentration for the bulk phase separation in case $1-3$ is $\eta_{\mathrm{M}}^{*} \sim 0.017$ ). As a result, only a minor shift of $\Phi_{\mathrm{LL}}$ is observed. However, as the surface separation $L=r-d_{\mathrm{L}}$ approaches a threshold value $L^{*} \sim d_{\mathrm{M}}, \Phi_{\mathrm{LL}}$ shows a sudden drop observed in Fig. 3 (b). As discussed in Sec. VII B, $L^{*}$ is the spinodal separation for a surface-induced phase transition ${ }^{19-21,37,38}$ and $\Phi_{\mathrm{LL}}$ calculated for $L<L^{*}$ should be discarded (see Sec. VII B for more details).

\section{B. Solvophilic surface and solvophobic solute (case 2)}

In the case labeled as case 2 the surface is solvophilic while the solute is solvophobic. Three subcases of case 2 (cases 2-1, 2-2, and 2-3) are considered and the parameters for these subcases are set as summarized in Table III. As observed in Fig. 4, although the surface interactions in cases 

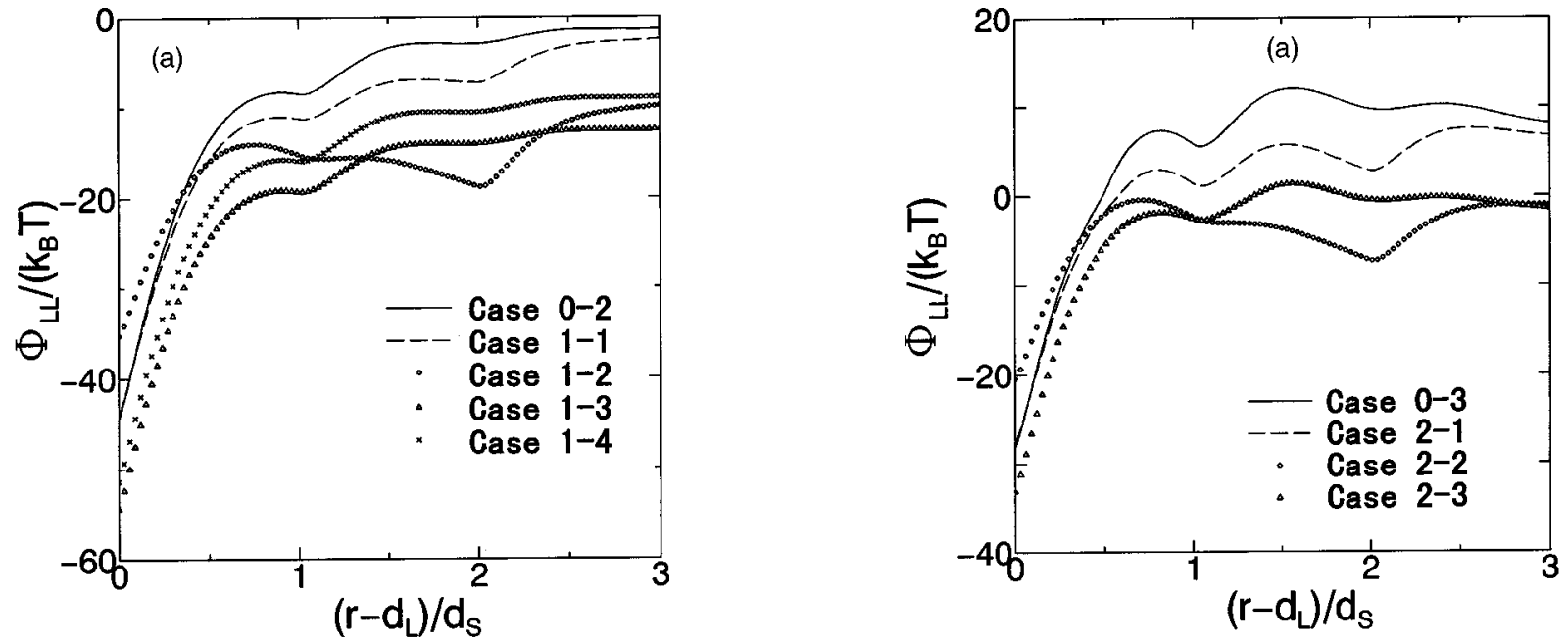

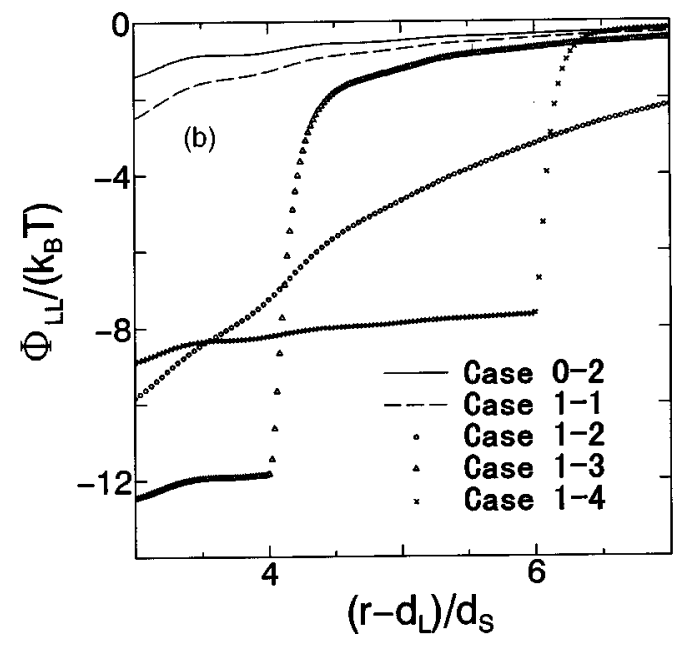

FIG. 3. Interaction between solvophobic surfaces immersed in solventsolute mixture: $0 \leqslant\left(r-d_{\mathrm{L}}\right) / d_{\mathrm{S}} \leqslant 3$ (a) and $3 \leqslant\left(r-d_{\mathrm{L}}\right) / d_{\mathrm{S}} \leqslant 7$ (b). The solute has considerably high solvophobicity. See Table II for the definition of cases $1-1,1-2,1-3$, and 1-4. In case $0-2$, no solute is added to the solvent.

0-2 and 0-3 are quite different, effects due to the solute addition for these cases are qualitatively similar with respect to the shift of the surface interaction $\Phi_{\mathrm{LL}}$. When the solute particles with $d_{\mathrm{M}}=2 d_{\mathrm{S}}$ are added to the solvent, $\Phi_{\mathrm{LL}}$ exhibits a downward shift that is minor under the lower concentration of the solute (case 2-1) but considerably large under the higher concentration (case 2-2). In case 2-3 where the highly solvophobic solute is present, $\Phi_{\mathrm{LL}}$ shows a sudden drop as $L \rightarrow L^{*} \sim d_{\mathrm{M}}$ [Fig. 4(b)].

TABLE III. Parameters set in the three subcases of case 2: cases 2-1, 2-2, and 2-3 (in case 2, the surface is solvophilic and the solute is solvophobic). The common parameters to all of these subcases are $\eta_{\mathrm{S}}+\eta_{\mathrm{M}}=0.383$, $4 \beta \varepsilon_{\mathrm{SS}}=1.0, \varepsilon_{\mathrm{MM}}=\varepsilon_{\mathrm{MS}}, \lambda_{\mathrm{LS}}=0.5, \kappa_{\mathrm{LS}}=5.0$, and $\lambda_{\mathrm{LM}}=0.0$.

\begin{tabular}{cccc}
\hline \hline Case & $d_{\mathrm{M}} / d_{\mathrm{S}}$ & $\eta_{\mathrm{M}}$ & $4 \beta \varepsilon_{\mathrm{MS}}$ \\
\hline $2-1$ & 2.0 & 0.010 & 0.0 \\
$2-2$ & 2.0 & 0.050 & 0.0 \\
$2-3$ & 4.0 & 0.001 & 0.0 \\
\hline \hline
\end{tabular}

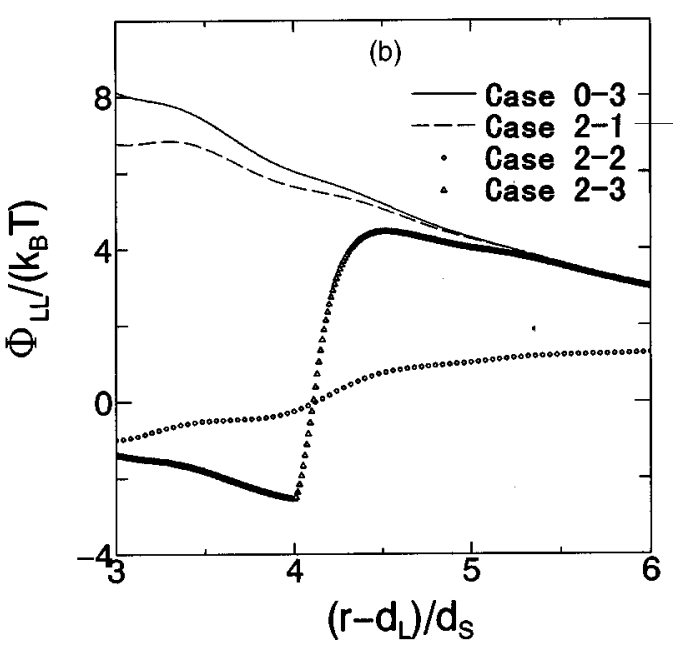

FIG. 4. Interaction between solvophilic surfaces immersed in solvent-solute mixture: $0 \leqslant\left(r-d_{\mathrm{L}}\right) / d_{\mathrm{S}} \leqslant 3$ (a) and $3 \leqslant\left(r-d_{\mathrm{L}}\right) / d_{\mathrm{S}} \leqslant 6$ (b). The solute has considerably high solvophobicity. See Table III for the definition of cases $2-1,2-2$, and 2-3. In case $0-3$, no solute is added to the solvent.

\section{Solvophobic surface and solvophilic solute (case 3)}

Let us turn our attention to a solute with considerably high solvophilicity. In the case labeled as case 3 the surface is solvophobic but the solute is solvophilic. Five subcases of case 3 (cases 3-1, 3-2, 3-3, 3-4, and 3-5) are considered and the values given to the parameters for these subcases are collected in Table IV. Effects of the solute addition are illustrated in Fig. 5. In the present model, as $d_{\mathrm{M}}$ increases with

TABLE IV. Parameters set in the five subcases of case 3: cases 3-1, 3-2, 3-3, $3-4$, and 3-5 (in case 3, the surface is solvophobic and the solute is solvophilic). The common parameters to all of these subcases are $\eta_{\mathrm{S}}+\eta_{\mathrm{M}}$ $=0.383,4 \beta \varepsilon_{\mathrm{SS}}=1.0, \varepsilon_{\mathrm{MM}}=\varepsilon_{\mathrm{MS}}, \lambda_{\mathrm{LS}}=0.0$, and $\lambda_{\mathrm{LM}}=0.0$.

\begin{tabular}{cccc}
\hline \hline Case & $d_{\mathrm{M}} / d_{\mathrm{S}}$ & $\eta_{\mathrm{M}}$ & $4 \beta \varepsilon_{\mathrm{MS}}$ \\
\hline $3-1$ & 2.0 & 0.050 & 1.0 \\
$3-2$ & 4.0 & 0.001 & 1.0 \\
$3-3$ & 4.0 & 0.050 & 1.0 \\
$3-4$ & 6.0 & 0.050 & 1.0 \\
$3-5$ & 6.0 & 0.001 & 0.7 \\
\hline \hline
\end{tabular}



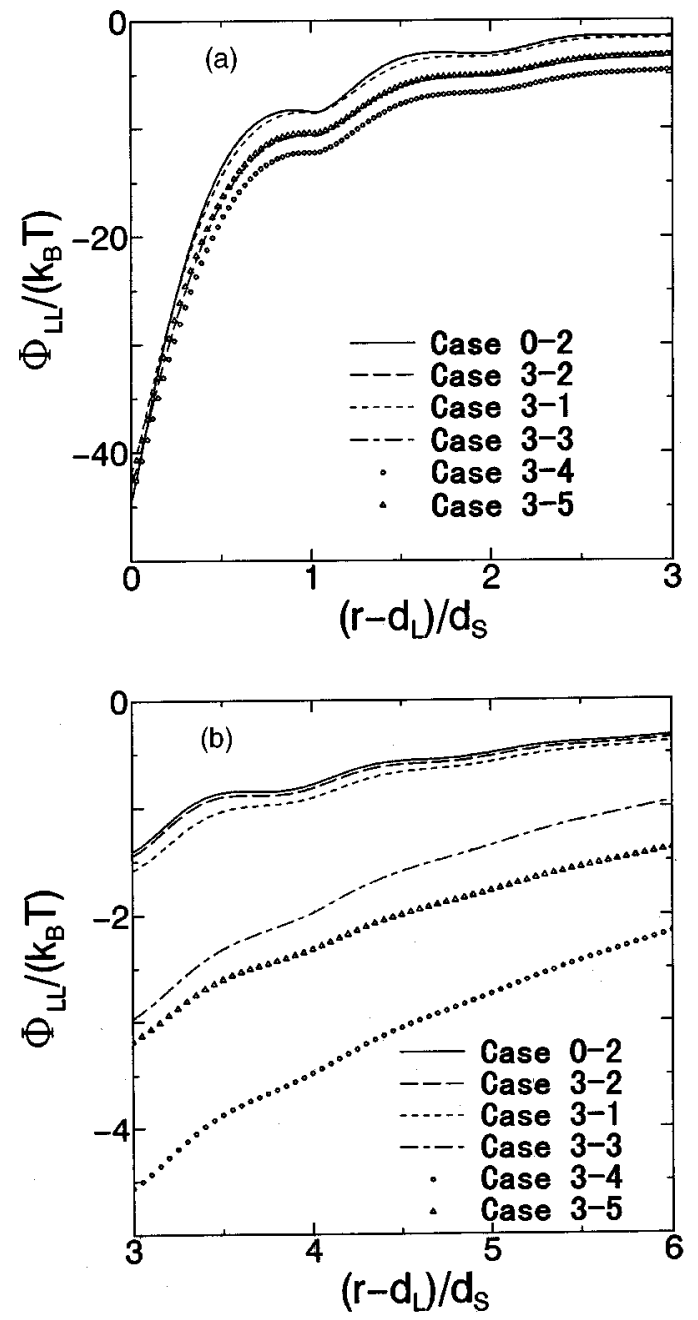

FIG. 5. Interaction between solvophobic surfaces immersed in solventsolute mixture: $0 \leqslant\left(r-d_{\mathrm{L}}\right) / d_{\mathrm{S}} \leqslant 3$ (a) and $3 \leqslant\left(r-d_{\mathrm{L}}\right) / d_{\mathrm{S}} \leqslant 6$ (b). The solute has considerably high solvophilicity. See Table IV for the definition of cases $3-1,3-2,3-3,3-4$, and 3-5. In case $0-2$, no solute is added to the solvent.

$\varepsilon_{\mathrm{MS}}$ fixed at a sufficiently large value (e.g., $4 \beta \varepsilon_{\mathrm{MS}}=1.0$ ), the solvation free energy becomes lower with the result of higher solvophilicity of the solute. When $\varepsilon_{\mathrm{MS}}$ is set at a smaller value with $d_{\mathrm{M}}$ unchanged, the solute becomes less solvophilic. The addition always causes a downward shift of the surface interaction. An increase in the solute solvophilicity or in the solute concentration leads to a more pronounced shift and a longer interaction range. For example, cases 3-1, 3-3, and 3-4 share the same $\eta_{\mathrm{M}}$ value, but the shift is the smallest in case 3-1 and the largest in case 3-4. The solute size is the same in cases 3-2 and 3-3, but the shift is larger in the latter case.

\section{Solvophilic surface and solvophilic solute (case 4)}

The case where both the surface and the solute are solvophilic is labeled as case 4 . Five subcases of case 4 (cases 4-1, 4-2, 4-3, 4-4, and 4-5) are considered and the parameters for these subcases are set as summarized in Table V. As shown in Fig. 6, the solute effects on the surface interaction $\Phi_{\mathrm{LL}}$ observed are qualitatively similar to those in cases of solvophobic surfaces in the sense that the solute addition
TABLE V. Parameters set in the five subcases of case 4: cases 4-1, 4-2, 4-3, 4-4, and 4-5 (in case 4, both the surface and the solute are solvophilic). The common parameters to all of these subcases are $\eta_{\mathrm{S}}+\eta_{\mathrm{M}}=0.383,4 \beta \varepsilon_{\mathrm{SS}}$ $=1.0, \varepsilon_{\mathrm{MM}}=\varepsilon_{\mathrm{MS}}, \lambda_{\mathrm{LS}}=0.5, \kappa_{\mathrm{LS}}=5.0, \lambda_{\mathrm{LM}}=0.5$, and $\kappa_{\mathrm{LM}}=5.0$.

\begin{tabular}{cccc}
\hline \hline Case & $d_{\mathrm{M}} / d_{\mathrm{S}}$ & $\eta_{\mathrm{M}}$ & $4 \beta \varepsilon_{\mathrm{MS}}$ \\
\hline $4-1$ & 2.0 & 0.050 & 1.0 \\
$4-2$ & 4.0 & 0.001 & 1.0 \\
$4-3$ & 4.0 & 0.050 & 1.0 \\
$4-4$ & 4.0 & 0.120 & 1.0 \\
$4-5$ & 6.0 & 0.050 & 1.0 \\
\hline \hline
\end{tabular}

always leads to a downward shift. The shift becomes more pronounced as the solute solvophilicity increases (case 4-1 $<$ case $4-3<$ case $4-5$ ) or the solute concentration becomes higher (case 4-2<case $4-3<$ case $4-4$ ). By comparing the results in cases 0-2 and 3-3 with those in cases 0-3 and 4-3, for example, one sees that the overall shift is larger in cases of solvophilic surfaces. All the interaction curves shown in Figs. 5 and 6 converge toward roughly the same value as the surface separation approaches zero (see the first paragraph of the next section).
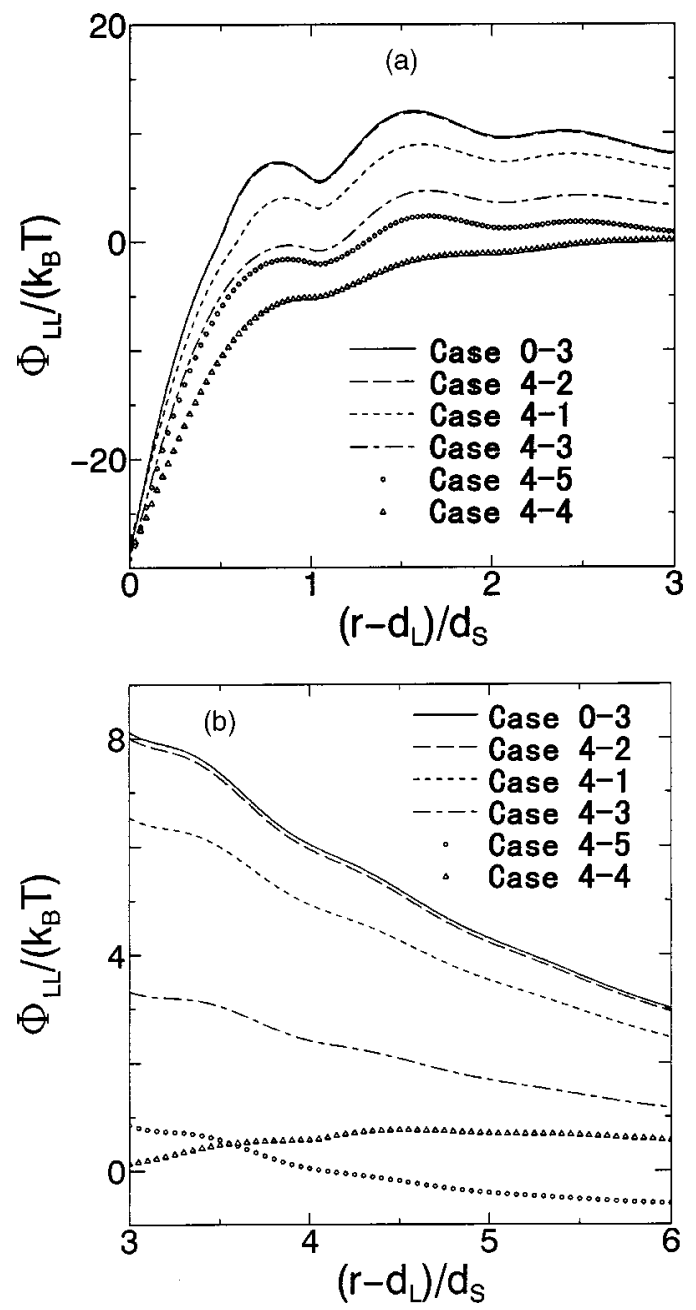

FIG. 6. Interaction between solvophilic surfaces immersed in solvent-solute mixture: $0 \leqslant\left(r-d_{\mathrm{L}}\right) / d_{\mathrm{S}} \leqslant 3$ (a) and $3 \leqslant\left(r-d_{\mathrm{L}}\right) / d_{\mathrm{S}} \leqslant 6$ (b). The solute has considerably high solvophilicity. See Table V for the definition of cases 4-1, $4-2,4-3,4-4$, and $4-5$. In case $0-3$, no solute is added to the solvent. 
TABLE VI. Parameters set in the two subcases of case 5: cases 5-1 and 5-2 (in case 5, all the small, medium-sized, and large spheres are hard ones with no attractions included in the potentials). The common parameters to all of these subcases are $\eta_{\mathrm{S}}+\eta_{\mathrm{M}}=0.383, \varepsilon_{\mathrm{SS}}=0.0, \varepsilon_{\mathrm{SM}}=0.0, \varepsilon_{\mathrm{MM}}=0.0, \lambda_{\mathrm{LS}}$ $=0.0$, and $\lambda_{\mathrm{LM}}=0.0$.

\begin{tabular}{ccc}
\hline \hline Case & $d_{\mathrm{M}} / d_{\mathrm{S}}$ & $\eta_{\mathrm{M}}$ \\
\hline $5-1$ & 4.0 & 0.001 \\
$5-2$ & 4.0 & 0.050 \\
\hline \hline
\end{tabular}

\section{E. Additional remarks}

As mentioned in Sec. II A, the total packing fraction of the solvent-solute mixture, $\eta_{\mathrm{S}}+\eta_{\mathrm{M}}$, is fixed at 0.383 . In a strict sense, it should be slightly smaller for a solvophobic solute and slightly larger for a solvophilic one. However, the author has verified that the fixing of the total packing fraction has only minor effects on the calculation result. Let us take cases 3-3 and 4-3, for instance, in which $\eta_{\mathrm{S}}$ is set at 0.333 . The surface interactions are calculated by increasing $\eta_{\mathrm{S}}$ by $5 \%\left(\eta_{\mathrm{S}}\right.$ is changed to 0.350$)$ with the other parameters kept constant $\left(\eta_{\mathrm{M}}=0.050\right)$. For case 3-3 the interaction $\Phi_{\mathrm{LL}}$ becomes more attractive for $\left(r-d_{\mathrm{L}}\right) / d_{\mathrm{S}}<1.3$ by less than $10 \%$. For larger separations it shifts in a more repulsive direction, but the change for $\left(r-d_{\mathrm{L}}\right) / d_{\mathrm{S}} \geqslant 3.0$ [see Fig. 5(b)] is less than $3 \%$. In case 4-3, $\Phi_{\mathrm{LL}}$ exhibits a downward shift for $\left(r-d_{\mathrm{L}}\right) / d_{\mathrm{S}}<0.35$ by less than $13 \%$. For larger separations it shifts in a more repulsive direction, but the maximum change for $\left(r-d_{\mathrm{L}}\right) / d_{\mathrm{S}} \geqslant 3.0$ [see Fig. 6(b)] is only about $8 \%$. Thus, the increase in $\eta_{\mathrm{S}}$ leads to no significant alteration of the calculation result, excepting the downward shift occurring even near the zero surface separation.

It is worthwhile to consider the case where all the small, medium-sized, and large spheres are hard ones with no attractions included in the potentials, which is labeled as case 5. Two subcases of case 5 (cases 5-1 and 5-2) are considered and the parameters for these subcases are set as summarized in Table VI. It has been found that the solute effects due to the entropic excluded volumes are much smaller. For instance, addition of medium-sized hard spheres to small hard spheres under the condition $d_{\mathrm{M}}=4 d_{\mathrm{S}}, \eta_{\mathrm{M}}=0.050$, and $\eta_{\mathrm{S}}$ $+\eta_{\mathrm{M}}=0.383$ causes no significant change in the interaction between large hard spheres (case 5-2) at the larger separations $\left(r-d_{\mathrm{L}}\right) / d_{\mathrm{S}} \geqslant 3$. The downward shift of $\Phi_{\mathrm{LL}}$ discussed above is caused by the attractive parts of the potentials. [With a much higher size asymmetry of the solvent-solute mixture $\left(d_{\mathrm{M}} \geqslant 10 d_{\mathrm{S}}\right)$, however, the entropic effects are substantially large even under a considerably low concentration of the medium-sized spheres. ${ }^{10}$ In fact, a sudden drop in the interaction between large hard spheres was reported in Ref. 10. Revisiting that behavior has revealed that the sudden drop represents the metastability limit for a surface-induced phase transition discussed in Sec. VII B, though it was interpreted in a different way in Ref. 10.]

\section{STRUCTURE OF BULK SOLVENT-SOLUTE MIXTURE}

Figure 7 shows the solute-solute pair distribution functions in the bulk mixture. In case 5-1 the structure of the

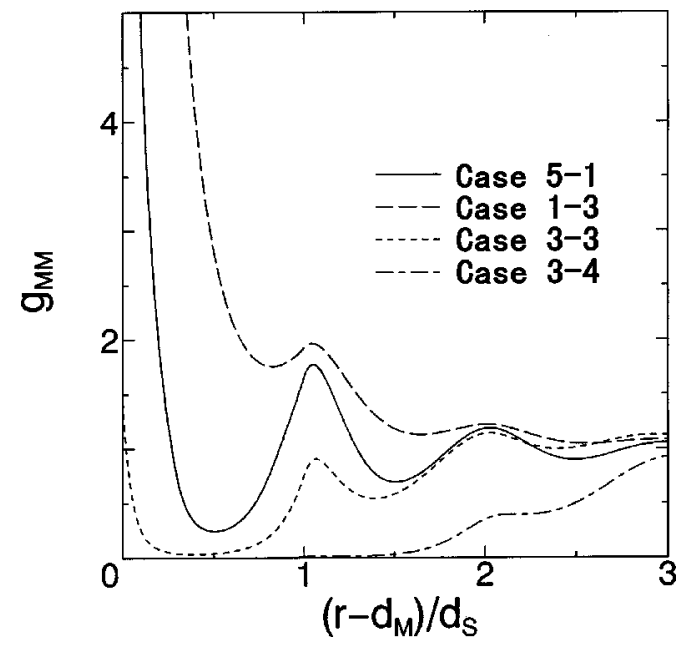

FIG. 7. Solute-solute pair distribution function in the bulk solvent-solute mixture. The solute is solvophobic in case 1-3 and solvophilic in cases 3-3 and 3-4. In case 5-1, the small and medium-sized spheres are both hard ones with no attractions included in the potentials. The values of $g_{\mathrm{MM}}$ at $\left(r-d_{\mathrm{M}}\right) / d_{\mathrm{S}}=0$ in cases 5-1 and 1-3 are, respectively, 15.4 and 68.8.

functions is determined from the entropic excluded-volume effects. Effects due to the attractive parts of the potentials are substantially large as evidenced by the results in the other cases. In is observed that solvophobic solute particles (case 1-3) form clusters while solvophilic ones (cases 3-3 and 3-4) are strongly solvated. The solvation is stronger for the solute with higher solvophilicity.

\section{STRUCTURE OF SOLVENT-SOLUTE MIXTURE AT A SINGLE SURFACE}

Figure 8 shows the reduced density profiles of solute particles near a single surface $g_{\mathrm{LM}}$ (the surface-solute pair distribution functions). In case 5-1 the structure of the profiles is determined by the entropic excluded-volume effects. In the other cases the attractive parts of the potentials play crucial roles in the profile formation. The solute is solvophobic in cases 1-3 and 2-3 and solvophilic in cases 3-3, 4-3, and 3-4. The surface is solvophobic in cases 1-3, 3-3, and $3-4$, and solvophilic surfaces are treated in cases 2-3 and 4-3. The qualitative characteristics of the profile are mainly determined from the solvent-solute affinity. Near a surface the solvophobic particles are enriched and the enrichment near the solvophobic surface is larger than near the solvophilic one. The enrichment becomes more pronounced as the solute solvophobicity increases. As for the solvophilic particles, they are depleted near a surface, and the depletion is more pronounced for the solute with higher solvophilicity. The contact values larger than unity in cases 3-3 and 4-3 are just remnants of the entropic effects. The depletion near the solvophilic surface is significantly more pronounced than near the solvophobic one.

Here, let us consider a solute particle at contact with the surface. The number of solvent particles surrounding the solute particle is about one-half of that around a solute particle in the bulk. It is reasonable that solvophobic solute particles are excluded from the bulk and come in contact with the surface by preference, leading to the formation of the en- 

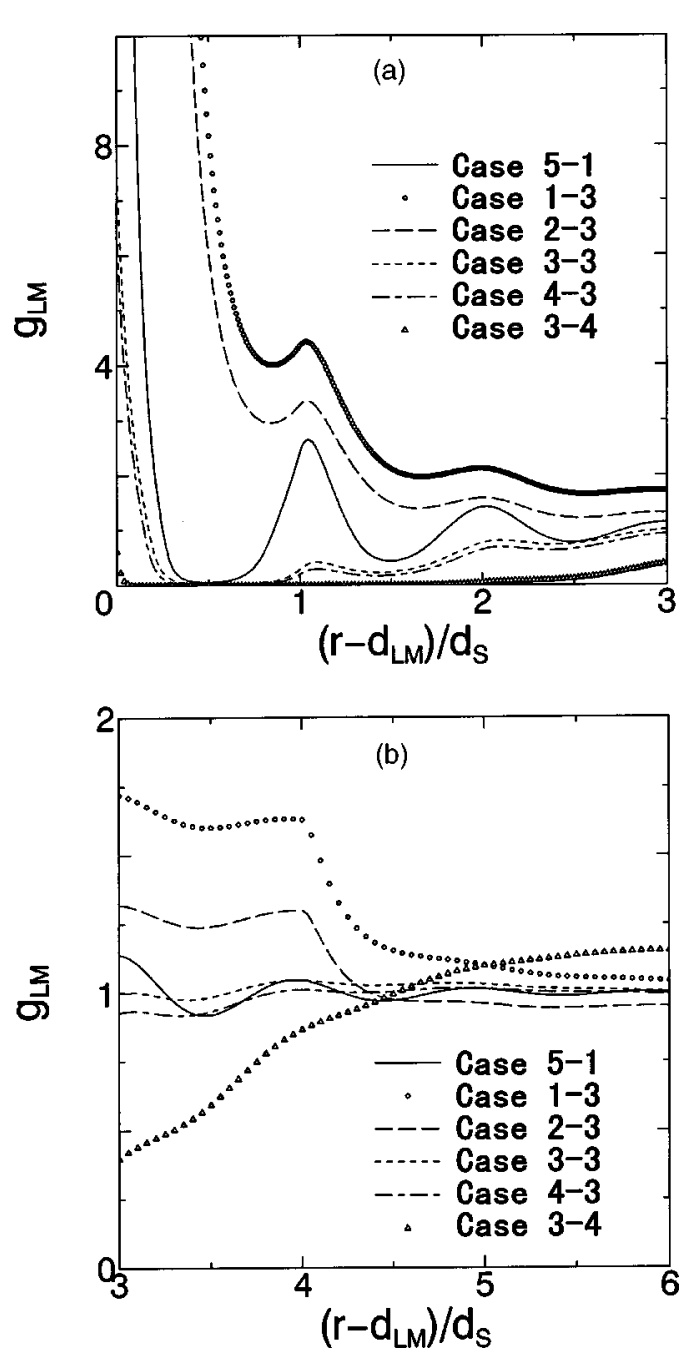

FIG. 8. Reduced density profile of solute particles (surface-solute pair distribution function) near a single surface: $0 \leqslant\left(r-d_{\mathrm{LM}}\right) / d_{\mathrm{S}} \leqslant 3$ (a) and 3 $\leqslant\left(r-d_{\mathrm{LM}}\right) / d_{\mathrm{S}} \leqslant 6(\mathrm{~b})$. The solute is solvophobic in cases 1-3 and 2-3 and solvophilic in cases 3-3, 4-3, and 3-4. The surface is solvophobic in cases 1-3, 3-3, and 3-4, and solvophilic surfaces are treated in cases 2-3 and 4-3. In case 5-1, all the small, medium-sized, and large spheres are hard ones with no attractions included in the potentials. The values of $g_{\mathrm{LM}}$ at $(r$ $\left.-d_{\mathrm{LM}}\right) / d_{\mathrm{S}}=0$ in cases $5-1,1-3$, and 2-3 are, respectively, 60.3, 1420, and 1040 .

riched layer. The entropic excluded-volume effects also cause enrichment (case 5-1), but these solvophobic effects are much larger. When the surface is made solvophilic, an additional factor arises: The solvent density near the solvophilic surface is higher than near the solvophobic one. With this factor the enrichment of solvophobic solute particles is somewhat reduced, but it is still considerably more pronounced than in case 5-1. When the surface solvophilicity is enhanced in cases $0-3$ and 2-3 by increasing $\beta \lambda_{\mathrm{LS}}$ to 1.0 with the other parameters unchanged, the surface interactions shift in considerably more repulsive directions. Furthermore, effects of the solvophobic solute on the surface interaction $\Phi_{\mathrm{LL}}$ become relatively smaller due to reduction of the solute enrichment near the surface.

Solvophilic solute particles, in contrast, wish to be strongly solvated in the bulk, and they are depleted near a solvophobic surface. In cases of a solvophilic surface, the solvent density near the surface is higher, giving rise to even more pronounced depletion of solute particles. Some additional calculations have been performed for case 4-3 using higher surface-solute affinity (up to $\beta \lambda_{\mathrm{LM}}=4.0$ ) with the other parameters unchanged ( $\beta \lambda_{L S}$ is fixed at 0.5 ). Nevertheless, both of the solute depletion near the surface and the downward shift of $\Phi_{\mathrm{LL}}$ persist. In conclusion, the structure formation of the solute particles near the surface is more pronounced in the combination where both the surface and the solute are solvophobic (Sec. IV A) or where both of them are solvophilic (Sec. IV D) than in the other two combinations (Sec. IV B and Sec. IV C).

\section{STRUCTURE OF SOLVENT-SOLUTE MIXTURE CONFINED BETWEEN TWO SURFACES}

\section{A. Effects due to surface separation}

Near a single surface solvophobic solute particles are enriched with the result that solvent particles are more depleted than in the case of pure solvent. This is particularly true near a solvophobic surface. In contrast, solvophilic solute particles prefer to stay away from the surface to be strongly solvated in the bulk, which holds even better near a solvophilic surface than near a solvophobic one. As two like surfaces approach each other, the behavior of the solvent and solute particles mentioned above becomes more conspicuous within the domain confined between two surfaces, particularly near the surfaces. Here, the discussion is limited to the surface separations $\left(r-d_{\mathrm{L}}\right) / d_{\mathrm{S}} \geqslant 2$ (see Sec. III). The information on the solvent-solute structure within the confined domain is contained in " $G_{\mathrm{LS}}-g_{\mathrm{LS}}$," " $G_{\mathrm{LM}}-g_{\mathrm{LM}}$," and $\Delta\left(G_{\mathrm{LS}}-g_{\mathrm{LS}}\right)$ defined in Sec. II B. These functions in cases 1-3 and 4-3 are shown in Figs. 9 and 10, respectively.

The concentration of the solvophobic solute within the confined domain becomes progressively higher as the solvophobic surfaces approach each other [Fig. 9(b)]. The solvent density decreases within the domain confined between two unfavorable surfaces, and further, significantly many solvent particles are excluded from the domain by the unfavorable solute particles entering it. The latter dominates because " $G_{\mathrm{LS}}-g_{\mathrm{LS}}$ " in Fig. 9(a) is almost equal to $\Delta\left(G_{\mathrm{LS}}-g_{\mathrm{LS}}\right)$ in Fig. 9(c). The solvent density within the domain reduces with decreasing $L$, and the reduction is more pronounced than in the pure-solvent case [Fig. 9(c)]. Since $\rho_{\mathrm{S}} \mid \Delta\left(G_{\mathrm{LS}}\right.$ $\left.-g_{\mathrm{LS}}\right) \mid$ is much larger than $\rho_{\mathrm{M}}\left(G_{\mathrm{LM}}-g_{\mathrm{LM}}\right)$, the depletion of solvent particles arising from the solute addition predominates over the enrichment of solute particles. This is also true in cases where the surface is solvophilic and the solute is solvophobic.

As two solvophilic surfaces approach each other, the solvent within the confined domain becomes denser and more packed [Fig. 10(a)]. The concentration of the solvophilic solute particles wishing to stay away from the surfaces, on the other hand, decreases to a significant extent [Fig. 10(b)]. As shown in Fig. 10(c), however, the increase in the solvent density near the surfaces within the confined domain with decreasing $L$ is smaller than in the pure-solvent case, except at small $L\left(L / d_{\mathrm{S}}<6\right)$ where there are essentially no solute particles left within the domain. [If the solvent particles be- 

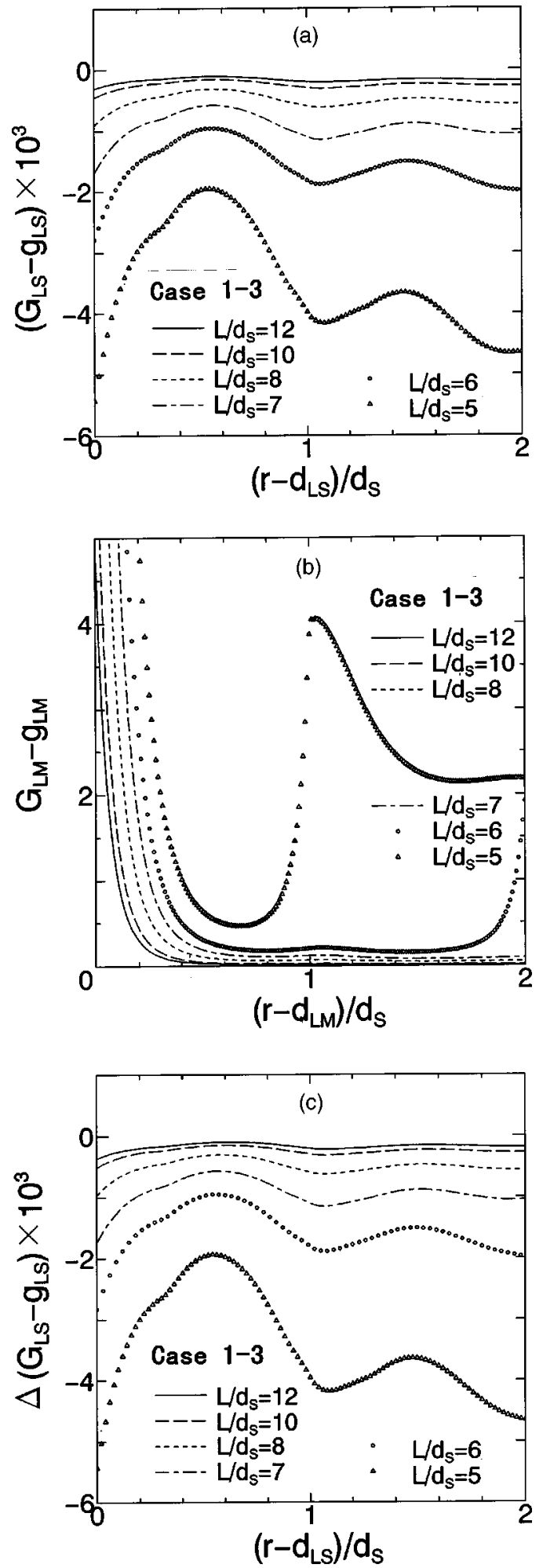

FIG. 9. Structure of solvent-solute mixture confined between two surfaces as a function of the surface separation in case 1-3: " $G_{\mathrm{LS}}-g_{\mathrm{LS}}$ " (a), " $G_{\mathrm{LM}}$ $-g_{\mathrm{LM}}$ " (b), and $\Delta\left(G_{\mathrm{LS}}-g_{\mathrm{LS}}\right)$ (c). In case 1-3, both the surface and the solute are solvophobic. The values of " $G_{\mathrm{LM}}-g_{\mathrm{LM}}$ " at $\left(r-d_{\mathrm{LM}}\right) / d_{\mathrm{S}}=0$ for $L / d_{\mathrm{S}}=10,8,7,6$, and 5 are, respectively, 7.18, 13.8, 26.0, 42.6, and 80.7.

come less packed within the confined domain due to the solute effects, $\Delta\left(G_{\mathrm{LS}}-g_{\mathrm{LS}}\right)$ becomes negative at the separations near $\left(r-d_{\mathrm{LS}}\right) / d_{\mathrm{S}} \sim n(n=0,1, \ldots)$ and positive at the other separations. In Fig. 10(c), negative $\Delta\left(G_{\mathrm{LS}}-g_{\mathrm{LS}}\right)$ oc-
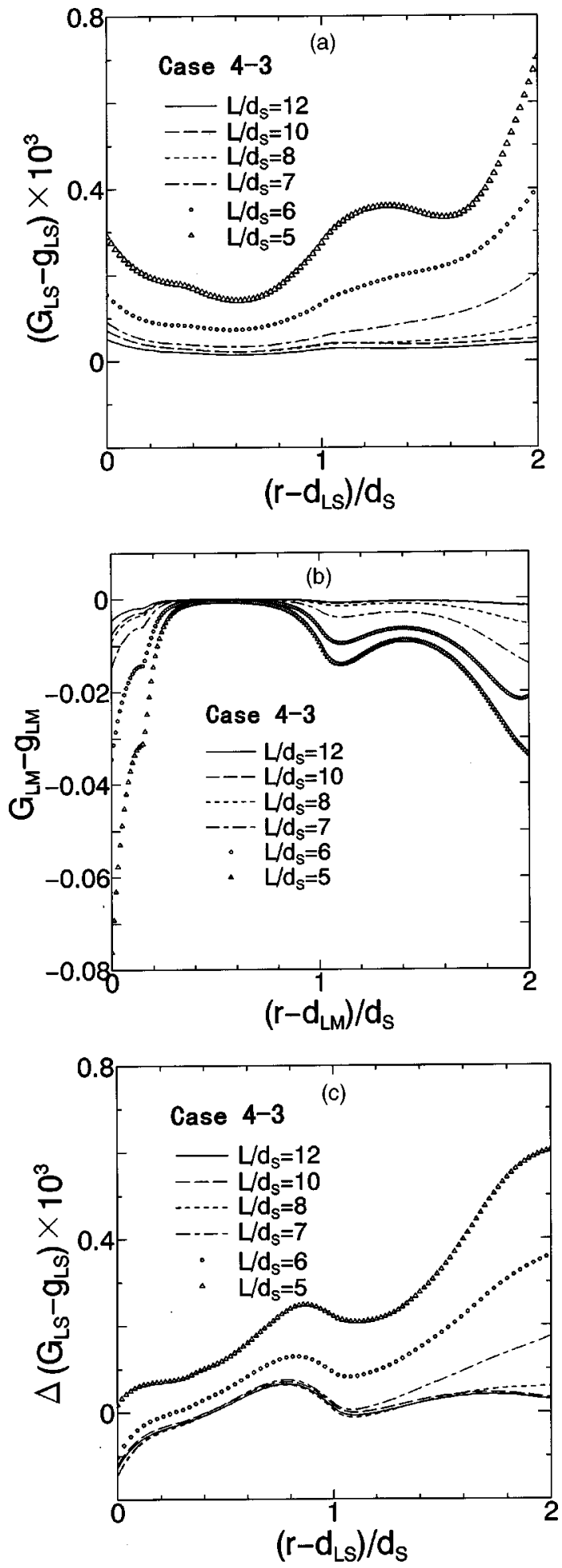

FIG. 10. Structure of solvent-solute mixture confined between two surfaces as a function of the surface separation in case 4-3: " $G_{\mathrm{LS}}-g_{\mathrm{LS}}$ " (a), " $G_{\mathrm{LM}}$ $-g_{\mathrm{LM}}$ " (b), and $\Delta\left(G_{\mathrm{LS}}-g_{\mathrm{LS}}\right)$ (c). In case $4-3$, both the surface and the solute are solvophilic.

curs only at $\left(r-d_{\mathrm{LS}}\right) / d_{\mathrm{S}} \sim 0$ and 1 . This means that the increase in the solvent density becomes smaller only near the surfaces.] This can be interpreted as follows. A highly solvophilic solute particle is strongly solvated. Since the solvation is quite stable, it persists while the solute particle moves away from the surfaces of the confined domain. The solvent 

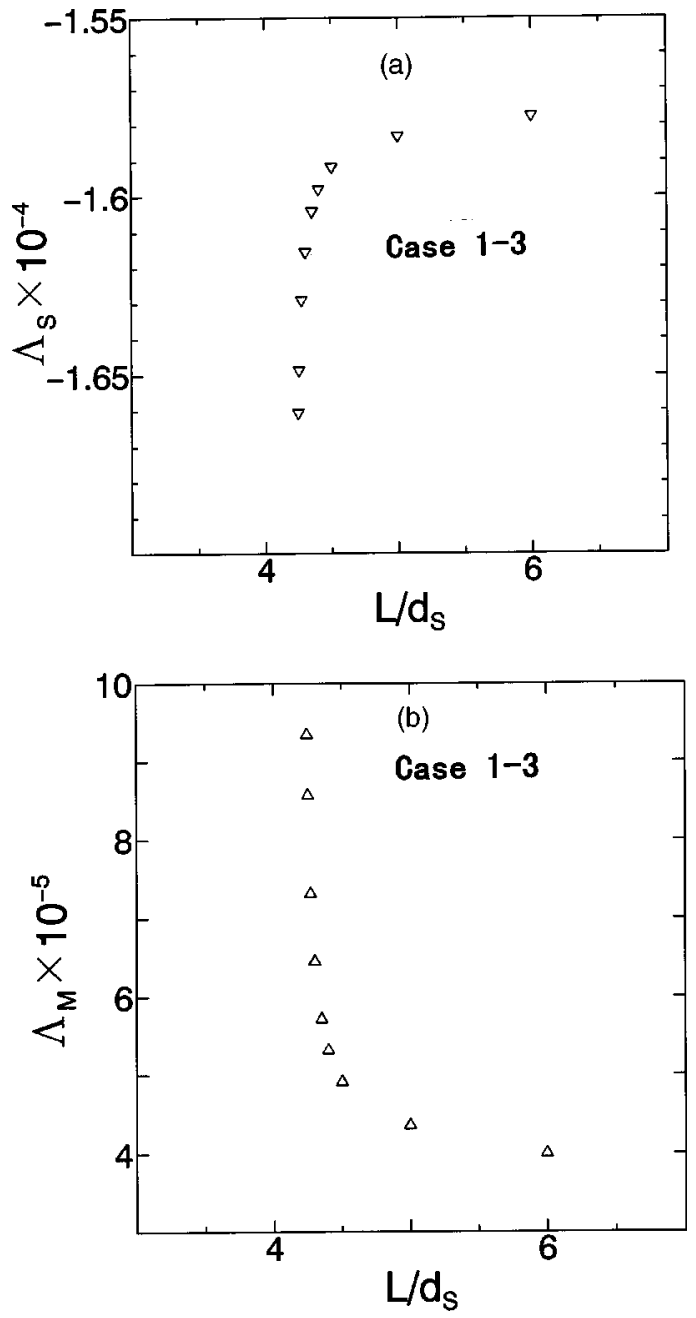

FIG. 11. Relation between $\Lambda_{i}(i=\mathrm{S}, \mathrm{M})$ and the surface separation $L$ in case 1-3: $i=\mathrm{S}$ (a) and $i=\mathrm{M}(\mathrm{b})$. In case 1-3, both the surface and the solute are solvophobic.

particles solvating the solute particle (i.e., those within the first solvation shell) also move away from the surfaces, leading to the smaller increase in the solvent density near the surfaces than in the pure-solvent case.

\section{B. Surface-induced phase transition}

To explore the sudden drop of the surface interaction $\Phi_{\mathrm{LL}}$ observed in Figs. 3(b) and 4(b), the relation between $\Lambda_{i}$ $(i=\mathrm{S}, \mathrm{M})$ and $L$ is examined in case 1-3 and the result is shown in Fig. 11. As $L \rightarrow L^{*} \sim d_{\mathrm{M}}, \Lambda_{\mathrm{S}}$ and $\Lambda_{\mathrm{M}}$ exhibit a sharp decrease and a sharp increase, respectively: The behavior of $\Lambda_{S} \rightarrow-\infty$ and $\Lambda_{M} \rightarrow+\infty$ is observed. Similar behavior was already found by the author ${ }^{19-21}$ and interpreted as a signal of wetting of the solute followed by drying of the solvent. The wetting-drying phenomenon is a surfaceinduced phase transition and $L^{*}$ is the spinodal separation. For $L \leqslant L^{*}$ the solvent-solute mixture confined by the surfaces cannot exist as a single phase even in a metastable state. Before the transition the number density of solvent particles within the domain is much higher than that of solute particles, though the latter is orders of magnitude higher than the bulk number density (it should be noted that the solute concentration in the bulk is very low). After the transition the domain confined between two surfaces is filled with the second phase of the solute. Since $L^{*} \sim d_{\mathrm{M}}$ in case 1-3, it is suggested that at $L \sim d_{\mathrm{M}}$ the domain is always filled with a monolayer of solute particles. As the solute concentration or the solute solvophobicity increases, $L^{*}$ becomes larger. When $\eta_{\mathrm{M}}$ is increased to 0.004 (this is still much lower than the bulk spinodal value $\sim 0.017$ ) in case $1-3$ with the other parameters unchanged, for instance, $L^{*} \sim 3 d_{\mathrm{M}}$. A further increase in $\eta_{\mathrm{M}}$ causes progressively larger $L^{*}$. Here $L^{*}$ is the metastability limit, and the transition followed by appearance of the second phase occurs at $L=L^{+}>L^{*}$ where the force becomes discontinuous with an abrupt drop. $L^{*}$ can be far larger than $d_{\mathrm{S}}$, leading to a long-range attractive interaction between solvophobic surfaces.

Recently, the interpretation of the author for the surfaceinduced phase transition was proved to be valid by Greberg and Patey ${ }^{37}$ using a grand canonical Monte Carlo (GCMC) computer simulation for a similar model system. They showed that $L^{+}$can be very large, leading to a long-range attractive interaction between like surfaces. It was also shown that even a trace amount of solute can cause dramatic effects. Their results are in qualitatively good accord with the theoretical predictions of the author in earlier works. ${ }^{19-21}$ The thermodynamic theory by Evans and Marini Bettolo Marconi ${ }^{38}$ and the experimental evidence reported by Christenson et al. ${ }^{39-41}$ also support these results.

It was shown in earlier works ${ }^{19-21}$ that $\Phi_{\mathrm{LL}}$ exhibits a sudden drop toward a negative, divergently large value as $L \rightarrow L^{*}$. As described in Sec. II B, $\Phi_{\mathrm{LL}}$ in the present study is calculated by treating a single surface immersed in the solvent-solute mixture. In such calculations the structure and properties of the mixture confined between two surfaces cannot be captured. In case $1-3$, for example, $L^{*} \sim d_{\mathrm{M}}$ is to be interpreted as the spinodal separation leading to the divergent behavior of $\Phi_{\mathrm{LL}}$, and the curve for $L<L^{*}$ should be discarded. Since the transition phenomenon is not the main subject in the present article, it is not pursued further (the readers should refer to Refs. $17-21$ and 37-41 for more details).

\section{PHYSICAL INTERPRETATION OF SOLUTE EFFECTS ON SURFACE INTERACTION}

The surface interaction is closely related to the structure of the solvent-solute mixture confined between two surfaces. In cases of solvophobic surfaces immersed in pure solvent, an important factor comes into play in addition to the entropic excluded-volume effects: The solvent is driven to be more depleted within the confined domain (particularly near the surfaces) as two surfaces approach each other. This factor is enhanced by the solute addition, though the mechanism of the enrichment for the solvophobic solute is different from that for the solvophilic solute. For the former the depletion is enhanced due to the enrichment of solute particles. For the latter, on the other hand, solute particles move away from the surfaces of the confined domain together with the solvent particles solvating them, leading to the enhancement of the depletion. 
As two solvophilic surfaces approach each other in pure solvent, the solvent is driven to be more enriched within the confined domain (particularly near the surfaces). This enrichment is reduced by the solute addition. When the solvophobic solute is added, solute particles are enriched within the domain, excluding solvent particles from the domain and causing the reduction of the enrichment of solvent particles. When the solvophilic solute is added, on the other hand, solute particles move away from the surfaces of the confined domain together with the solvent particles solvating them, leading to the reduction of the enrichment of solvent particles. As the solvophilicity of the surface increases, the solviphobic-solute effects become smaller while the solvophilic-solute effects larger.

Thus, the solute addition causes either enhancement of the depletion or reduction of the enrichment of solvent particles within the domain confined between two surfaces (particularly near the surfaces). As a result, the thermal pressure due to the solvent particles, which contributes to the mean force as a repulsive component, becomes lower than in the pure-solvent case. In the presence of solvophobic solute, the enrichment of solute particles within the confined domain generates thermal pressure due to the solute particles, giving rise to an additional contribution to the repulsive component. As described above, however, the depletion of solvent particles caused by the solute addition predominates over the enrichment of solute particles [i.e., $\rho_{\mathrm{S}}\left|\Delta\left(G_{\mathrm{LS}}-g_{\mathrm{LS}}\right)\right|$ is much larger than $\left.\rho_{\mathrm{M}}\left(G_{\mathrm{LM}}-g_{\mathrm{LM}}\right)\right]$, and the repulsivecomponent decrease arising from the lower thermal pressure due to the solvent particles dominates. Thus, the solute addition leads to a relatively more attractive net force. In cases of solvophilic solute, the solute concentration is much higher than in cases of solvophobic solute, but the solute particles are depleted near the surfaces. Hence, the contribution from the thermal pressure due to the solute particles remains rather small. In conclusion, when the solute is added to the solvent, the net force becomes relatively more attractive and the interaction (potential of mean force) exhibits a downward shift. It is interesting that the surface interaction $\Phi_{\mathrm{LL}}$ always shifts in a more attractive direction once a solute with sufficiently high solvophobicity or solvophilicity is added to the solvent. (At very small surface separations a minor upward shift of $\Phi_{\mathrm{LL}}$ is observed in some cases.) The solute effects become larger as the solute solvophobicity or solvophilicity increases and the solute concentration becomes higher.

The solute effects are in all likelihood the smallest when the solute is neither solvophobic nor solvophilic. To check this conjecture, additional calculations are performed for some different values of $\varepsilon_{\mathrm{MS}}$ with the other parameters kept constant: $\quad \eta_{\mathrm{M}}=0.05, \quad d_{\mathrm{M}}=4 d_{\mathrm{S}}, \quad \eta_{\mathrm{S}}+\eta_{\mathrm{M}}=0.383, \quad 4 \beta \varepsilon_{\mathrm{SS}}$ $=1.0, \varepsilon_{\mathrm{MM}}=\varepsilon_{\mathrm{MS}}, \lambda_{\mathrm{LS}}=0.0$, and $\lambda_{\mathrm{LM}}=0.0$ (the surface is always solvophobic). The results are shown in Fig. 12. Overall, the solute effects are the smallest for $4 \beta \varepsilon_{\mathrm{MS}}=0.50$ among the five values tested. The interaction in the case of $4 \beta \varepsilon_{\mathrm{MS}}=0.50$ is more attractive than in case $0-2$ at some separations and more repulsive at other separations but the differences are rather small. The solute with $4 \beta \varepsilon_{\mathrm{MS}}$ higher than 0.50 causes a downward shift of $\Phi_{\mathrm{LL}}$ and the shift becomes larger with an increase in the solute solvophilicity.
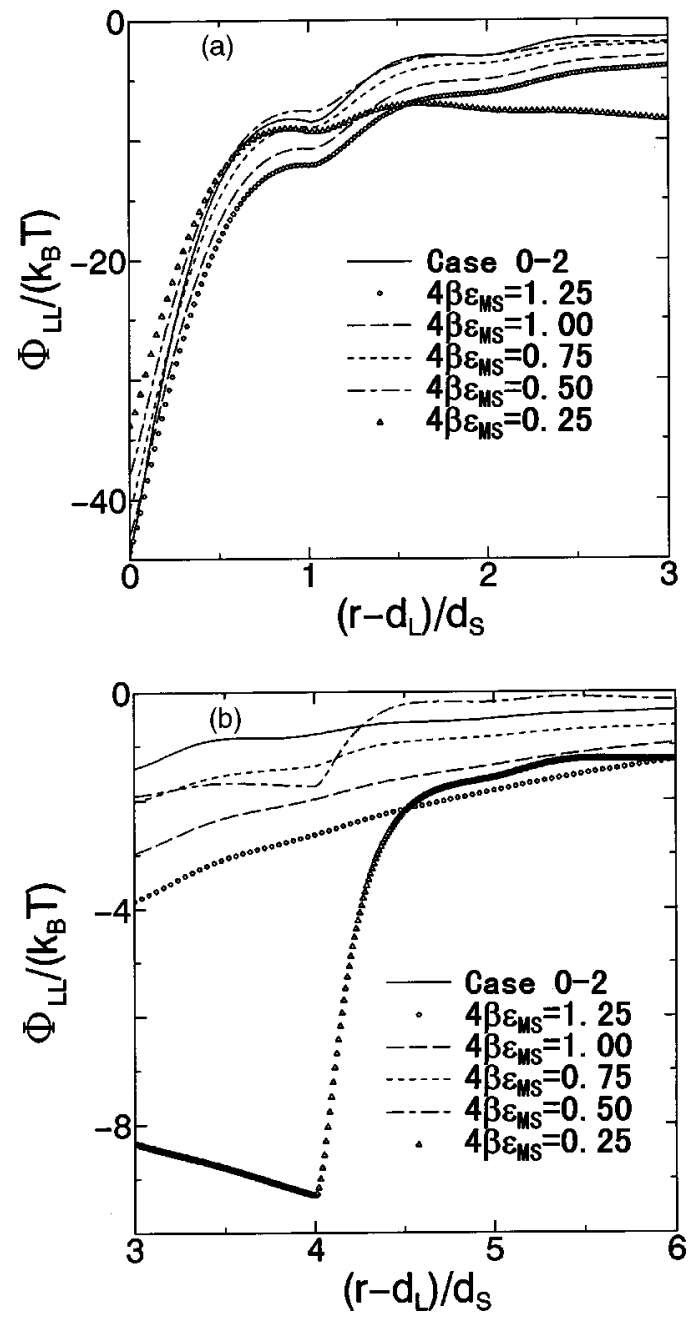

FIG. 12. Interaction between solvophobic surfaces immersed in solventsolute mixture: $0 \leqslant\left(r-d_{\mathrm{L}}\right) / d_{\mathrm{S}} \leqslant 3$ (a) and $3 \leqslant\left(r-d_{\mathrm{L}}\right) / d_{\mathrm{S}} \leqslant 6$ (b). Effects due to the solvent-solute affinity $\varepsilon_{\mathrm{MS}}$. In case $0-2$, no solute is added to the solvent. The common parameters to all the plots except in case 0-2 are $\eta_{\mathrm{M}}=0.05, d_{\mathrm{M}}=4 d_{\mathrm{S}}, \eta_{\mathrm{S}}+\eta_{\mathrm{M}}=0.383,4 \beta \varepsilon_{\mathrm{SS}}=1.0, \varepsilon_{\mathrm{MM}}=\varepsilon_{\mathrm{MS}}, \lambda_{\mathrm{LS}}=0.0$, and $\lambda_{\mathrm{LM}}=0.0$ (the surface is always solvophobic).

The solute with $4 \beta \varepsilon_{\mathrm{MS}}=0.25$ is solvophobic and $\Phi_{\mathrm{LL}}$ in its presence is characterized by the sudden drop at $L \sim d_{\mathrm{M}}$ discussed above.

\section{CONCLUSION}

Solute effects on the solvent-induced interaction between surfaces have been analyzed using integral equation theories with bridge functions incorporated in the closure equations. A simple model of spherical particles is chosen to roughly mimic hydrophobic or hydrophilic surfaces immersed in water containing hydrophobic or hydrophilic solute. The singlet $\mathrm{OZ}$ approach is adopted to calculate the structure of the solvent-solute mixture near a single surface and the surface interaction induced. The RISM theory ${ }^{22}$ is also utilized to analyze the structure of the mixture confined between two surfaces as a function of the surface separation. The semiempirical method of estimating the bridge functions, which was pioneered by Verlet ${ }^{28}$ and further developed by Duh and Henderson, ${ }^{33}$ has been extended to a system 
comprising particles with extremely high size asymmetry (large spheres immersed in a mixture of small and mediumsized spheres).

In cases of solvophobic surfaces immersed in pure solvent, an important factor arises in addition to the entropic excluded-volume effects: As two surfaces approach each other, the solvent is driven to be more depleted within the domain confined between surfaces (particularly near the surfaces). In contrast, within the domain confined between two solvophilic surfaces in pure solvent, the solvent is driven to be more enriched (particularly near the surfaces), and the enrichment becomes larger with a decrease in the surface separation. Solute addition to the solvent causes either enhancement of the depletion (in cases of solvophobic surfaces) or reduction of the enrichment (in cases of solvophilic surfaces) of solvent particles within the confined domain. This is true for both solvophobic and solvophilic solutes. The mechanism of the enhancement or the reduction for a solvophobic solute is different from that for a solvophilic solute. Solvophobic solute particles are enriched within the confined domain, excluding the solvent particles from the domain. Solvophilic particles, on the other hand, move away from the surfaces of the domain together with the solvent particles solvating them. These enhance the depletion or reduce the enrichment. Overall, the solute effects are more pronounced in the combination where both the surface and the solute are solvophobic or where both of them are solvophilic than in the other two combinations.

The enhancement of the depletion or the reduction of the enrichment of solvent particles near the surfaces within the confined domain leads to lower thermal pressure due to the solvent particles and a smaller contribution to the repulsive component of the net force induced between surfaces. In the presence of solvophobic solute, the enrichment of solute particles within the confined domain generates thermal pressure due to the solute particles, giving rise to an additional contribution to the repulsive component. As described above, however, the depletion of solvent particles caused by the solute addition predominates over the enrichment of solute particles, and the repulsive-component decrease arising from the lower thermal pressure due to the solvent particles dominates. Thus, the solute addition leads to a relatively more attractive net force with the result that the surface interaction exhibits a downward shift. It is interesting to note that the surface interaction always shifts in a more attractive direction once a solute with sufficiently high solvophobicity or solvophilicity is added to the solvent. The solute effects become larger as the solute solvophobicity or solvophilicity increases and the downward shift can be remarkable even under a low solute concentration. With lower solvophobicity or solvophilicity of the solute, a higher solute concentration is required to make the effects sufficiently large. The effects are the smallest when the solute is neither solvophobic nor solvophilic.

Suppose that solute molecules larger than water molecules are added to a system where macromolecules are immersed in aqueous solution. There are significantly many experimental observations ${ }^{42,43}$ suggesting that the forces between macromolecules are made remarkably more attractive by the solute addition. One often takes the view that the macromolecules generate excluded volumes for the solute molecules added and attractive forces are entropically induced between macromolecules (or equivalently, the forces between macromolecules become more attractive) ${ }^{42,43}$ However, the physical origins of the induced forces are not that simple as shown in the present study. The effective forces are determined by complicated interplay of the water-water, water-solute, solute-solute, water-macromolecule, and solute-macromolecule interaction potentials, and the attractive parts of the potentials play crucial roles.

In the protein folding, the attractive interaction between hydrophobic portions of a protein molecule is of vital importance. The interaction is referred to as the hydrophobic interaction. When the hydrophobic interaction is attributable to the reorganization of water structure near the hydrophobic portion, however, it is moderately strong and short range, reaching only several water diameters. ${ }^{15,16}$ In the presence of solute molecules with significantly high hydrophobicity or hydrophilicity (these are usually larger than water molecules), the attractive interaction is strengthened and made longer range. If the surface-induced phase transition occurs, powerful attractions arise. Note that protein folding is promoted not in pure water but in aqueous solution containing a variety of solute molecules with hydrophobicity and hydrophilicity. The author believes that these effects play essential roles in accelerating the protein folding.

\section{ACKNOWLEDGMENTS}

The force induced between large hard spheres immersed in small hard spheres obtained in the computer simulation of T. Biben, P. Bladon, and D. Frenkel was smoothed and integrated by R. Roth, R. Evans, and S. Dietrich. The resultant data, which were given to the author from them, is compared with the theoretical result in Fig. 1. The author wishes to express his sincere thanks to these researchers.

${ }^{1}$ P. Attard, J. Chem. Phys. 91, 3083 (1989).

${ }^{2}$ P. Attard and G. N. Patey, J. Chem. Phys. 92, 4970 (1990).

${ }^{3}$ M. Kinoshita, S. Iba, K. Kuwamoto, and M. Harada, J. Chem. Phys. 105, 7177 (1996).

${ }^{4}$ B. Götzelmann, R. Evans, and S. Dietrich, Phys. Rev. E 57, 6785 (1998).

${ }^{5}$ R. Roth, B. Götzelmann, and S. Dietrich, Phys. Rev. Lett. 83, 448 (1999).

${ }^{6}$ Y. Rosenfeld, Phys. Rev. Lett. 63, 980 (1989).

${ }^{7}$ R. Roth, R. Evans, and S. Dietrich, Phys. Rev. E 62, 5360 (2000).

${ }^{8}$ M. Kinoshita and T. Oguni, Chem. Phys. Lett. 351, 79 (2002).

${ }^{9}$ M. Kinoshita, J. Chem. Phys. 116, 3493 (2002).

${ }^{10}$ M. Kinoshita, Chem. Phys. Lett. 353, 259 (2002).

${ }^{11}$ P. Attard, D. Wei, G. N. Patey, and G. M. Torrie, J. Chem. Phys. 93, 7360 (1990).

${ }^{12}$ P. Attard, D. R. Bérard, C. P. Ursenbach, and G. N. Patey, Phys. Rev. A 44, 8224 (1991)

${ }^{13}$ S. Amokrane, J. Chem. Phys. 108, 7459 (1998).

${ }^{14}$ J. Clément-Cottuz, S. Amokrane, and C. Regnaut, Phys. Rev. E 61, 1692 (2000).

${ }^{15}$ M. Kinoshita and F. Hirata, J. Chem. Phys. 104, 8807 (1996).

${ }^{16}$ M. Kinoshita, S. Iba, and M. Harada, J. Chem. Phys. 105, 2487 (1996).

${ }^{17}$ M. Kinoshita, Mol. Phys. 94, 485 (1998).

${ }^{18}$ M. Kinoshita, Mol. Phys. 96, 71 (1999).

${ }^{19}$ M. Kinoshita, Chem. Phys. Lett. 325, 281 (2000).

${ }^{20}$ M. Kinoshita, Chem. Phys. Lett. 326, 551 (2000).

${ }^{21}$ M. Kinoshita, Chem. Phys. Lett. 333, 217 (2001).

${ }^{22}$ D. Chandler and H. C. Andersen, J. Chem. Phys. 57, 1930 (1972).

${ }^{23}$ Y. Rosenfeld and N. W. Ashcroft, Phys. Rev. A 20, 1208 (1979). 
${ }^{24}$ P. H. Fries and G. N. Patey, J. Chem. Phys. 82, 429 (1985).

${ }^{25}$ D. R. Bérard and G. N. Patey, J. Chem. Phys. 95, 5281 (1991).

${ }^{26}$ F. Lado, E. Lomba, and M. Lombardero, J. Chem. Phys. 103, 481 (1995).

${ }^{27}$ M. Alvarez, F. Lado, E. Lomba, M. Lombardero, and C. Martín, J. Chem. Phys. 107, 4642 (1997).

${ }^{28}$ L. Verlet, Mol. Phys. 41, 183 (1980).

${ }^{29}$ S. Labík, A. Malijevský, and W. R. Smith, Mol. Phys. 73, 495 (1991).

${ }^{30}$ R. Pospísil, A. Malijevský, S. Labík, and W. R. Smith, Mol. Phys. 74, 253 (1991).

${ }^{31}$ S. Labík, A. Malijevský, R. Pospísil, and W. R. Smith, Mol. Phys. 74, 261 (1991).

${ }^{32}$ E. Lomba, C. Martín, M. Lombardero, and J. A. Anta, J. Chem. Phys. 96, 6132 (1992).

${ }^{33}$ D.-M. Duh and D. Henderson, J. Chem. Phys. 104, 6742 (1996).
${ }^{34}$ T. Biben, P. Bladon, and D. Frenkel, J. Phys.: Condens. Matter 8, 10799 (1996).

${ }^{35}$ B. V. Derjaguin, Kolloid-Z. 69, 155 (1934).

${ }^{36}$ R. Dickman, P. Attard, and V. Simonian, J. Chem. Phys. 107, 205 (1997).

${ }^{37}$ H. Greberg and G. N. Patey, J. Chem. Phys. 114, 7182 (2001).

${ }^{38}$ R. Evans and U. Marini Bettolo Marconi, J. Chem. Phys. 86, 7138 (1987).

${ }^{39}$ H. K. Christenson, J. Colloid Interface Sci. 104, 234 (1985).

${ }^{40}$ H. K. Christenson and C. E. Blom, J. Chem. Phys. 86, 419 (1987).

${ }^{41}$ H. K. Christenson, J. Fang, and J. N. Israelachvili, Phys. Rev. B 39, 11750 (1989)

${ }^{42}$ S. B. Zimmerman and A. P. Minton, Annu. Rev. Biophys. Biomol. Struct. 22, 27 (1993).

${ }^{43}$ A. P. Minton, Curr. Opin. Biotechnol. 8, 65 (1997). 